\title{
Antibacterial activity of eight dentine bonding systems
}

\author{
Monika Łukomska-Szymańska ${ }^{1}$, Beata Zarzycka ${ }^{2}$, Jolanta Żurawska- \\ Olszewska ${ }^{2}$, Violetta Olbert-Sieroszewska ${ }^{1}$, Krzysztof Sokołowski ${ }^{3}$, Zbigniew \\ Krzemiński ${ }^{2}$, Jerzy Sokołowski ${ }^{1}$ \\ ${ }^{1}$ Department of General Dentistry, Medical University of Lodz, Poland \\ ${ }^{2}$ Department of Clinical Immunology and Microbiology, Medical University of Lodz, Poland \\ ${ }^{3}$ Department of Conservative Dentistry, Medical University of Lodz, Poland
}

\begin{abstract}
Unchecked bacterial invasion of dentine leads to inflammatory pulp disease, root canal infection, and periapical disease. Enterococcus faecalis and Streptococcus mutans play an important role in these outcomes. The aim of the study was to investigate the antibacterial properties of Syntac Classic, Optibond FL, XP Bond, Bond 1, NRC, Clearfil Protect Bond, Bond 1 SF and Solist against S. mutans and E. faecalis. Antibacterial properties were tested by means of diffusion method with $0.2 \%$ chlorhexidine being used for comparative purposes. It was found that bonding systems possess different degrees of antibacterial activity. Clearfil Protect Bond and Syntac Classic exhibited some antibacterial activity while Heliobond/Syntac Classic, Bond Clearfil Protect Bond and Adhesive Optibond FL did not. The antibacterial properties of a dentine bonding system depended on the $\mathrm{pH}$ value.
\end{abstract}

Key words: antibacterial agents, antimicrobial tests, dental adhesive

\section{INTRODUCTION}

Unchecked bacterial invasion of dentine leads to inflammatory pulp disease, root canal infection, and periapical disease. Enterococcus faecalis and Streptococcus mutans play an important role in these outcomes.

$E$. faecalis is associated with different forms of periradicular disease, including primary endodontic infections and persistent infections [1]. It was found in $24 \%$ to $77 \%$ of root-filled teeth with periradicular lesions [1-6], while failed endodontic treatment cases are nine times more likely to contain E. faecalis than primary infections [1]. E. faecalis manages to survive in the root canal system in many ways. It shows widespread genetic polymorphisms [7], it binds to dentin by the secretion of serine protease, gelatinase, and collagen-binding protein (Ace) [8], its small size enables successful invasion and proliferation within dentinal tubules [9] and it is able to survive for a long time without nutrition [10]

S. mutans, being the leading cause of dental caries, is considered to be the most cariogenic of all the oral streptococci. The cariogenicity of this microorganism is based on the metabolism of sucrose, which is a substrate for extracellular and membrane-bound glucosyltransferases and an efficient growth substrate for $S$. mutans. The abilities of $S$. mutans to adhere firmly to tooth surfaces in the presence of sucrose and to form acids by fermenting various dietary sugars have been associated with its caries-inducing potential $[9,11,12]$.

There are two possible mechanisms initiating the formation of secondary caries. Firstly, resin polymerisation shrinkage causes gaps between the restoration and the cavity walls that can be colonized by oral microorganisms from saliva [13-15]. Secondly, incomplete caries removal can be another source of microbes. Although minimally invasive dentistry puts pressure on dentists to avoid cavity over-preparation there are no instruments or materials available that can objectively verify the presence or absence of bacteria in the prepared floor of a cavity [16-18]. The application of a bonding system with antibacterial activity is a promising solution. Several dentin bonding systems are currently available. The traditional bonding systems demand enamel etching and the application of either a primer and bond ( $4^{\text {th }}$ generation) or only a primer/bond $\left(5^{\text {th }}\right.$ generation). However, the newer generations are self-etch primers/adhesives which no longer demand orthophosphoric acid usage. The sixth generation selfetch adhesives can be either two-step (self-etch primer and bond) or one-step (self-etch adhesive, primer A+B). Procedures all-in-one self-etch adhesives constitute the $7^{\text {th }}$ and $8^{\text {th }}$ generations (solvent-free). Since there are many different bonding systems on the market, and companies are still introducing new ones, it is crucial to know which bonding systems possess the best clinical performance. The success rate of such a restorative procedures relies strictly on the antibacterial activity of the bonding system employed.

The purpose of this study was to determine the effect of various bonding systems on Streptococcus mutans and Enterococcus faecalis. 


\section{Materials and Methods}

Eight commercially available bonding systems were investigated (Table 1).

\subsection{Agar diffusion assay - glass cylinder}

The antibacterial properties of these systems against Streptococcus mutans ATCC 25175 and Enterococcus faecalis ATCC 29212 were determined according to the recommendations of CLSI. Overnight culture of these organism in Brain Heart Infusion Broth (Oxoid) was adjusted to 0.5 turbidity according to the McFarland scale and placed on Mueller-Hinton Agar (Oxoid) plates using sterile swabs. Next, an automatic pipette (Lab Mate) was used to add $6 \mu$ of each bonding system (for a 2- or 3-stage system, each component was added in equal proportions up to $6 \mu \mathrm{l}$ ) to a sterile glass cylinder $10 \mathrm{~mm}$ in diameter and $8 \mathrm{~mm}$ in height in the agar plate(Fig. 1) and polymerized by means of Elipar ${ }^{\mathrm{TM}}$ S10 LED Curing Lamp, (3M ESPE) according to the manufacturer's instructions. The plates were incubated at a temperature of $35^{\circ} \mathrm{C}$ for 48 hours in an aerobic atmosphere enriched with $\mathrm{CO}_{2}\left(\mathrm{CO}_{2}\right.$ Gen, Oxoid $)$. After incubation, the diameters of the inhibition zones around the cylinders were measured (Fig. 2). A $0.2 \%$ aqueous solution of chlorhexidine was used as a positive control. Each bonding system was examined five times.

\subsection{Statistical procedures}

A statistical analysis was conducted to confirm the statistical significance of any differences,. As the data was unbalanced and abnormally distributed, the non-parametric Kruskal-Wallis rank test was performed, followed by a multiple-comparison test in order to test the differences in pairs. A significance level of $p<0.05$ was assumed. In order to estimate any dependence of a bonding system's $\mathrm{pH}$ upon the growth arrest area's diameter, a robust regression method was performed.

\section{Results}

The antibacterial activity of the samples is shown in Figure 3. A significant difference was found in the antibacterial activity of the bonding systems investigated. The following statistical significances were found; for Streptococcus mutans ( $\mathrm{p}<0.001$ for the general model):

- Primer Clearfil Protect Bond versusOptiBond FL,

- Primer Syntac Classic versus CHX,

- Primer Syntac Classic versusClearfil Protect Bond,

- Primer Syntac Classic versus Primer Clearfil Protect Bond,

- Syntac Classic versus CHX,

- Syntac Classic versus Primer Clearfil Protect Bond,

And for Enterococcus faecalis ( $<<0.001$ for the general model):

- Syntac Classic versus Primer Syntac Classic,

- Syntac Classic versus Solist,

- Primer Syntac Classic versus CHX,

- Primer Syntac Classic versus Primer Clearfil Protect Bond,

- $\mathrm{NRC}$ versus $\mathrm{CHX}$,

- Solistversus CHX.

The antibacterial effect against both Streptococcus mutans and_Enterococcus faecalis of the bonding systems tested was inversely proportional to the $\mathrm{pH}$ value (statistical significance; $\mathrm{p}<0.001$ ) (Figs. 4,5).

\section{Discussion}

The agar disk-diffusion test has been widely accepted as a simple screening method to assess the antibacterial properties of dentin bonding systems $[18,19]$. After being applied to a disc, the investigated material is placed on agar plates and inoculated with oral bacteria. If the material releases any antibacterial components, an inhibition halo is produced. The strength of antibacterial activity is measured by the diameter of the inhibition zone around the material.

In the present study, a modified agar diffusion method was employed. In this, glass cylinders were used instead of absorbent paper discs to replicate clinical conditions more closely because the application of a two- or more-component bonding system on an absorbent paper disc may lead to the formation of layers in the disc. Moreover, since the penetration of bonding agents is strictly associated with their viscosity [17], $4^{\text {th }}$ generation primers and, probably, the $5^{\text {th }}$ generation bonding systems will penetrate to the full depth of an absorbent paper disc. Cylinders also guarantee a strictly limited and replicable area of material application, allowing direct contact between all the components of the adhesive system and the agar, exactly as in the case of adhesive resin application on the dentine surface. Moreover, although halogen light (and supposedly light from LED source) are incapable of passing through a paper disc impregnated with the adhesive components, this is not the case for cylinders [17]. 
The size of the inhibition zone depends on the antibacterial properties of the materials, the quantity used, and the diffusion potential across the culture medium. The greater the quantity and the higher the diffusion potential, the larger the inhibition zones which can be observed. While the quantity of material applied can be easily controlled thanks to use of an automatic pipette, the final result may be influenced by the diffusion potential, as it is closely related to the chemical composition of the tested adhesive. However, as Turkun et al. [19] obtained similar results for both direct inhibition methods (agar well technique, paper disc technique, dentin disc technique) and tooth cavity model technique, it can be speculated that the diffusion potential does not have a major influence on the results of antibacterial properties studies.

Chlorhexidine was chosen as the control in order to make the studies comparable with the research of other authors. A literature review highlights a considerable discrepancy between the antibacterial properties of different adhesive systems. For example AdheSE Primer and Adper Prompt L-Pop showed a higher degree of inhibition for $S$. mutans than Clearfil Protect Bond Primer and Futurabond NR, but a lower one than Cervitec (1\% CHX) [20]. By contrast, Clearfil Protect Bond was found to exhibited the greatest effective antibacterial activity against oral streptococci, promoting inhibition zones significantly higher than those associated with $0.12 \%$ chlorhexidine for $S$. cricetus and $S$. oralis [21]. This statement is broadly in agreement with the present study (however the difference was not statistically significant) and with other investigations [19,22,23]. In our studies, three bonding agents (Heliobond/Syntac Classic, Clearfil Protect Bond and Optibond FL) exhibited no antibacterial activity, which could be due to their high $\mathrm{pH}$ value and lack of monomer.

The polymerizable monomer, methacryloyloxydodecylpyridinium bromide (MDPB), a constituent of Primer Clearfil Protect Bond and synthesised from quaternary ammonium, exhibits strong bactericidal activity. Not only can it inactivate the residual bacteria in the cavity, but it can also inhibit the bacteria which invade through microleakage. In other words, antibacterial action takes place both before and after polymerization [24]. This is partially due to the capacity of MDPB to copolymerize with other resin monomers immobilised within the polymer matrix [17]. The MDPB molecule has a special configuration containing a positively-charged pyridinium group which can produce an electrical imbalance in the bacterial cell wall, causing cell wall destruction ultimately leading to bacterial death [25]. According to Imazato et al. [24,26]_unpolymerised MDPB shows strong bactericidal activity and is able to inactivate residual bacteria. Another advantage of MDPB incorporation is increase in the hydrophobicity of the adhesive, which may result in the bonding interface having higher hydrolytic stability [27]. Our study confirms the strong bactericidal activity of the MDPB-containing Primer Clearfil Protect Bond.

Although the bonding agent of Clearfil Protect Bond contains NaF crystals, it exhibits no antibacterial activity. However, both the fluoride-containing One-up Bond F Plus (not photo-activated) and One-up Bond F (light-cured) exhibit a strong antibacterial effect [21,28]. This difference may be explained by the fact that the fluoride in Clearfil Protect Bond remains incorporated within the polymer matrix after curing, hence preventing fluoride release into solution.

Previously, dentin bonding systems with low $\mathrm{pH}$ were reported to have superior antibacterial properties [26,29]. However, recent studies demonstrate little or no effect of $\mathrm{pH}$ value on bacterial growth [18,30]. In another study, in which Clearfil Protect Bond with and without MDPB was investigated, the authors concluded that the antibacterial effect of Clearfil Protect Bond depends mainly on the presence of MDPB and only slightly on the acidity of the material_[31]. However our study does not support their opinion as we found that the antibacterial effect does relay on the $\mathrm{pH}$ value.

\section{Conclusions}

Within the limitations of this in vitro study, the following conclusions can be drawn:

1. Bonding systems possess different degrees of antibacterial activity.

2. Primer Clearfil Protect Bond (against S. mutans) and Syntac Classic (againstE. faecalis) exhibit the best antibacterial activity of all the bonding systems tested.

3. The antibacterial properties of dentin bonding systems depend on their $\mathrm{pH}$ value.

\section{Acknowledgement}

We would like to thank Prof J.G. Shaw DDS (U.Birm) F.D.S.RCS (Eng.) for his assistance with the English script. 


\section{References}

[1]. I.N. Rôças, J.F. Siqueira and K.R.N. Santos, Association of Enterococcus faecalis with different forms of periradicular diseases, Journal of Endodontics,30(5), 2004, 315-20.

[2]. B.P.F.A. Gomes, E.T. Pinheiro, C.R. Gade-Neto, E.L.R. Sousa, C.C.R. Ferraz, A.A. Zaia, F.B. Teixeira_and_F.J. Souza-Filho, Microbiological examination of infected dental root canals,_Oral Microbiology and Immunology, 19(2), 2004, 71-76.

[3]. H.H. Hancock, A. Sigurdsson, M. Trope and_J. Moiseiwitsch, Bacteria isolated after unsuccessful endodontic treatment in a North American population,_Oral Surgery, Oral Medicine, Oral Pathology, Oral Radiology, and Endodontics, 91(5), 2001, 579586.

[4]. A. Molander, C. Reit, G. Dahlen_and_T. Kvist, Microbiological status of root-filled teeth with apical periodontitis, International Endodontic Journal, 31(1), 1998, 1-7.

[5]. J.F. SiqueiraandI. Rôças. Polymerase chain reaction-based analysis of microorganisms associated with failed endodontic treatment,_Oral Surgery, Oral Medicine, Oral Pathology, Oral Radiology, and Endodontics, 97(1), $2004,85-94$.

[6]. G. Sundqvist, D. Figdor, S. Persson_and_U. Sjogren, Microbiologic analysis of teeth with failed endodontic treatment and the outcome of conservative re-treatment,_Oral Surgery, Oral Medicine, Oral Pathology, Oral Radiology, and Endodontics, 85(1), 1998, 86-93.

[7]. C.M. Sedgley, S.L. Lennan and_D.B. Clewell, Prevalence, phenotype, and genotype of oral Enterococci,_Oral Microbiology and Immunology, 19(2), 2004, 95-101.

[8]. T.S. Hubble, J.F. Hatton, S.R. Nallapareddy, B.E. Murray and_M.J. Gillespie, Influence of Enteroccocus faecalis proteases and the collagen-binding protein, Ace, on adhesion to dentin,_Oral Microbiology and Immunology, 18(2), 2003, 121-126.

[9]. S. Hamada and_H.D. Slade, Biology, immunology, and cariogenicity of Streptococcus mutans,_Microbiological Reviews, 44(2), 1980, 331-384

[10]. D. Figdor, J.K. Davies and_G. Sundqvist, Starvation survival, growth and recovery of Enterococcus faecalis in human serum, Oral Microbiology and Immunology, 18(4), 2003, 234-239.

[11]. V.A. Lee, R. Karthikeyan, H.R. Rawls and_B.T. Amaechi, Anti-cariogenic effect of a cetylpyridinium chloride-containing nanoemulsion, Journal of Dentistry, 38(9), 2010, 742-749.

[12]. W.I. Loesche, Role of Streptococcus mutans in human dental decay,_Microbiological Reviews, 50(4), $1986,353-380$.

[13]. R.M. Browne and R.S. Tobias, Microbial microleakage and pulpal inflammation: a review, Endodontics \& dental traumatology,2(5), 1986,177-183.

[14]. B. Haller and A. Trojanski, Effect of multi-step dentin bonding systems and resin-modified glass-ionomer cement liner on marginal quality of dentin-bonded resin composite Class II restorations, Clinical Oral Investigations, 2(3), 1998,130-136.

[15]. I.A. Mjor, Frequency of secondary caries at various anatomical locations, Operative Dentistry, 10(3), 1985,88-92.

[16]. D.W. Boston and_H.T. Graver,_Histobacteriological analysis of acid red dye-stainable dentin found beneath intact amalgam restorations, Operative Dentistry, 19(2), 1994,65-69.

[17]. J.O. Gondim, C. Duque, J.Hebling_and_E.M. Giro, Influence of human dentine on the antibacterial activity of self-etching adhesive systems against cariogenic bacteria, Journal of Dentistry, 36(4), 2008,241-248.

[18]. M. Vaidyanathan,_E.C._Sheehy, S.C. Gilbert and_D. Beighton, Antimicrobial properties of dentine bonding agents determined using in vitro and ex vivo methods, Journal of Dentistry, 37(7), 2009,514-521.

[19]. L.S. Türkün, M. Ates, M. Türkün_and_E. Uzer, Antibacterial activity of two adhesive systems using various microbiological methods,_Journal of Adhesive Dentistry, 7(4),2005,315-320.

[20]. Y. Korkmaz, M. Ozalp and N. Attar, Comparison of the antibacterial activity of different self-etching primers and adhesives, Journal of Contemporary Dental Practice, 9(7),2008,57-64.

[21]. C.M. Esteves, C. Ota-Tsuzuki, A.F. Reis and_J.A. Rodrigues, Antibacterial activity of various self-etching adhesive systems against oral streptococci, Operative Dentistry, 35(4), 2010,448-453.

[22]. A. Karanika-Kouma, P. Dionysopoulos, E. Koliniotou-Koubia_and_A. Kolokotronis,Antibacterial properties of dentin bonding systems, polyacid-modified composite resins and composite resins,_Journal of Oral Rehabilitation, 28(2),2001,157-160.

[23]. M.M. Lobo, R.B. Gonçalves, LA Pimenta, A.K. Bedran-Russo and_P.N.Pereira, In vitro evaluation of caries inhibition promoted by self-etching adhesive systems containing antibacterial agents,_Journal of Biomedical Materials Research Part B: Applied Biomaterials, 75(1), 2005,122-127.

[24]. S. Imazato,_Antibacterial properties of resin composites and dentin bonding systems,_Dental Materials, 19(6), 2003,449-457.

[25]. http://www.protect-bond.com/en/

[26]. S. Imazato,_T._Imai and_S. Ebisu, Antibacterial activity of proprietary self-etching primers,_American Journal of Dentistry, 11(3), 1998,106-108.

[27]. S. Imazato, F.R. Tay, A.V. Kaneshiro, Y. Takahashi and_S. Ebisu, An in vivo evaluation of bonding ability of comprehensive antibacterial adhesive system incorporating MDPB,_Dental Materials, 23(2), 2007,170-176.

[28]. R. Walter, W.R. Duarte, P.N.R. Pereira, H.O. Heymann, E.J. Swift Jr and R.R. Arnold, In vitro inhibition of bacterial growth using different dental adhesive systems,_Operative Dentistry, 32(4), 2007,388-393.

[29]. M._Herreraa, P._Carrióna, M._Bravoa_and_A._Castillo, Antibacterial activity of four dentin bonding systemsInternational Journal of Antimicrobial Agents, 15(4), 2000,305-309.

[30]. G._Schmalz, Z._Ergücü_and_K.A._Hiller, Effect of dentin on the antibacterial activity of dentin bonding agents,_Journal of Endodontics, 30(5), 2004,352-358.

[31]. S. Imazato, A. Kuramoto, Y. Takahashi, S. Ebisu_and_M.C. Peters, In vitro antibacterial effects of the dentin primer of Clearfil Protect Bond,_Dental Materials, 22(6), 2006,527-532. 
Table 1 Features of tested bonding systems.

\begin{tabular}{|c|c|c|c|c|c|}
\hline $\begin{array}{c}\text { Adhesive } \\
\text { system }\end{array}$ & Manufacturer & Generation & Type & pH & Composition \\
\hline Syntac Classic & $\begin{array}{l}\text { IvoclarVivadent, } \\
\text { Schaan, Liechtenstein }\end{array}$ & $4^{\text {th }}$ & 3 stages & $\begin{array}{l}1.3 \\
4.0 \\
\text { not } \\
\text { determined } \\
\end{array}$ & $\begin{array}{l}\text { Primer: } \\
\text { Tetraethylenglycoldimethacrylate, } \\
\text { Maleic acid, Dimethylketone, Water } \\
\text { Adhesive: } \\
\text { Polyethylenglycoldimethacrylate, } \\
\text { Maleic acid, Glutaraldehyde, Water } \\
\text { Bond:Bis-GMA, dimethacrylate, } \\
\text { initiators and stabilizers }\end{array}$ \\
\hline Optibond FL & $\begin{array}{l}\text { Kerr, Orange, } \\
\text { CA, USA }\end{array}$ & $4^{\text {th }}$ & 3 stages & 1.9 & $\begin{array}{l}\text { FL Primer: HEMA, GPDM, MMEP, } \\
\text { water, ethanol, CQ, BHT } \\
\text { FL Adhesive: Bis-GMA, HEMA, } \\
\text { GDMA, CQ, ODMAB, filler (fumed } \\
\text { SiO2, barium aluminoborosilicat, } \\
\text { Na2SiF6), coupling factor A174 }\end{array}$ \\
\hline XP Bond & $\begin{array}{l}\text { DENTSPLY } \\
\text { De Trey, } \\
\text { Konstanz, } \\
\text { Germany }\end{array}$ & $5^{\text {th }}$ & 2 stages & 2.1 & $\begin{array}{l}\text { PENTA, TCB, HEMA, TEGDMA, } \\
\text { UDMA, tert-butanol, nanofiller, CQ, } \\
\text { stabilizer }\end{array}$ \\
\hline Bond 1 & $\begin{array}{l}\text { Pentron } \\
\text { Corporation, } \\
\text { Wallingford, } \\
\text { CT, USA }\end{array}$ & $5^{\text {th }}$ & 2 stages & 5.0 & $\begin{array}{l}\text { Adhesive: PMGDM, HEMA, } \\
\text { TMPTMA, initiators, acetone }\end{array}$ \\
\hline $\mathrm{NRC}$ & $\begin{array}{l}\text { DentsplyDeTrey, } \\
\text { Konstanz, Germany }\end{array}$ & $6^{\text {th }}$ & 2 stages & 1.2 & maleic and itaconic acids \\
\hline $\begin{array}{l}\text { Clearfil Protect } \\
\text { Bond }\end{array}$ & $\begin{array}{l}\text { KurarazDental Inc., } \\
\text { Kurashiki, Japan }\end{array}$ & $6^{\text {th }}$ & 2 stages & 2.0 & $\begin{array}{l}\text { Primer: MDPB, MDP, HEMA, } \\
\text { hydrophilic dimethacrylate, photo- } \\
\text { initiator, water } \\
\text { Bond: MDP, HEMA, Bis-GMA, } \\
\text { hydrophobic dimethacrylate, photo- } \\
\text { initiators, silanated colloidal silica, } \\
\text { surface-treated NaF }\end{array}$ \\
\hline Bond $1 \mathrm{SF}$ & $\begin{array}{l}\text { Pentron Clinical } \\
\text { Orange, CA, USA }\end{array}$ & $8^{\text {th }}$ & 1 stage & 3.0 & $\begin{array}{l}\text { UDMA, TEGDMA, HEMA, \& 4-MET } \\
\text { resins, silane-treated bariumborosilicate } \\
\text { glasses, silica with initiators, stabilizers } \\
\text { and UV absorber, organic and/or } \\
\text { inorganic pigments, opacifiers }\end{array}$ \\
\hline Solist & $\begin{array}{l}\text { DMG, } \\
\text { Hamburg, } \\
\text { Germany }\end{array}$ & $7^{\text {th }}$ & 1 stage & 2.2 & $\begin{array}{l}\text { Adhesive: HEMA, TEGDMA, } \\
\text { elastomers, methacrylated phosphoric } \\
\text { acid }\end{array}$ \\
\hline
\end{tabular}

Fig. 1 Bonding systems were added to sterile glass cylinders with an automatic pipette.

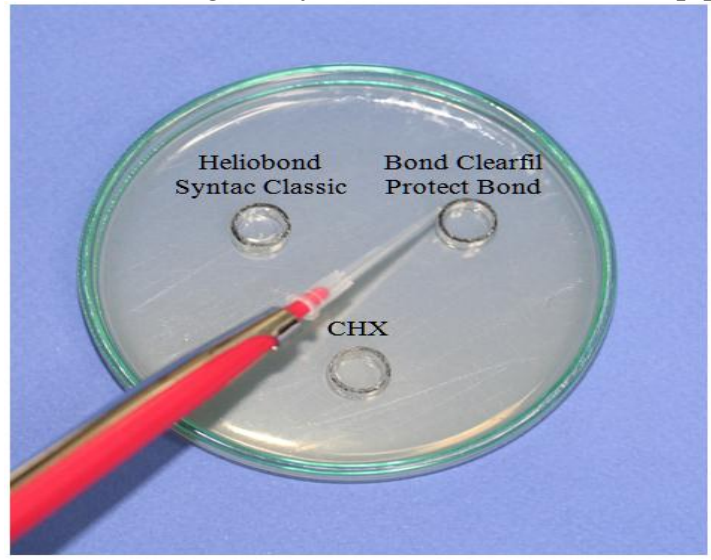


Fig. 2 Aafter incubation, the diameters of the inhibition zones were measured. $\mathrm{CHX}=\mathrm{Chlorhexidine.}$
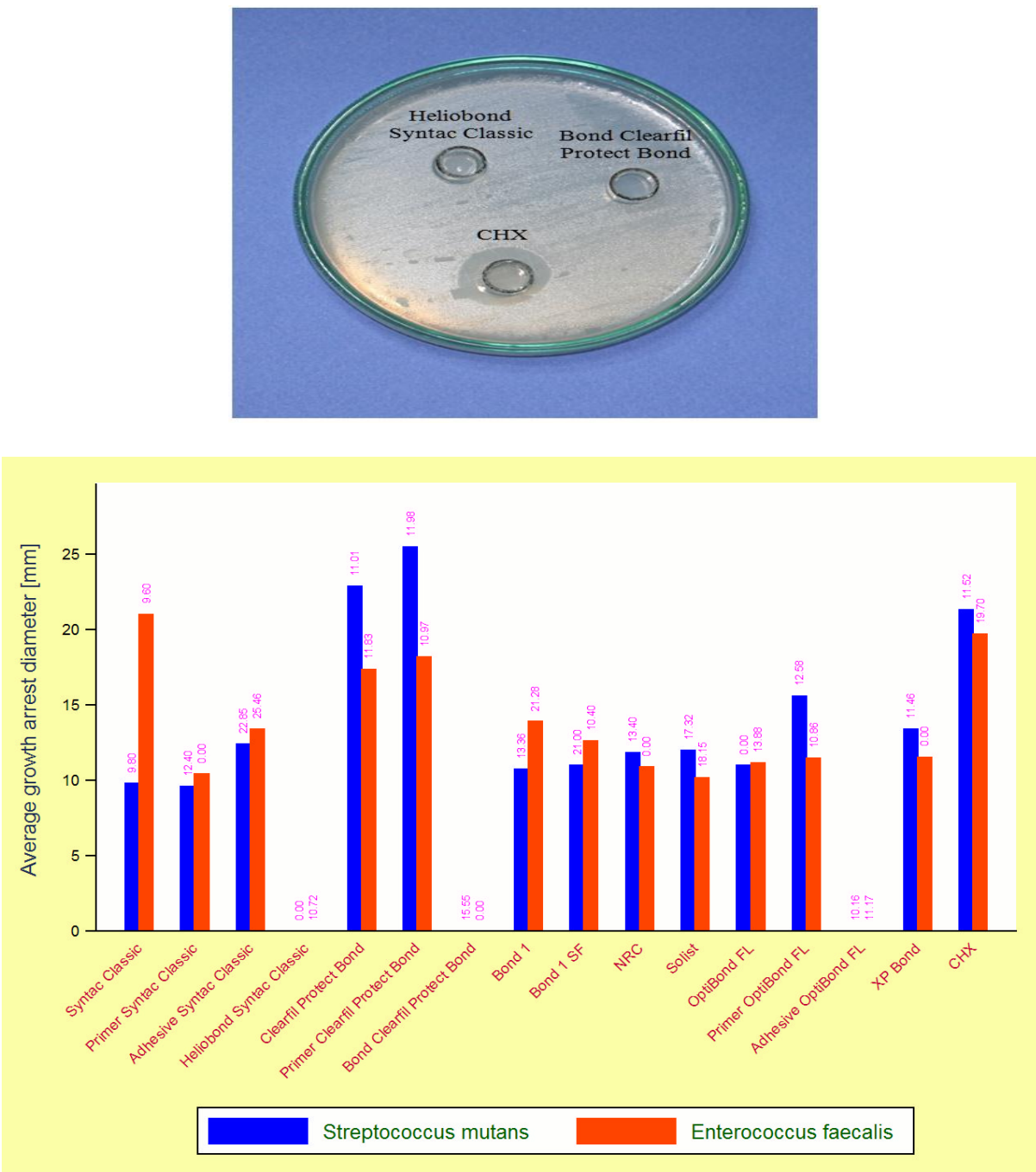

Fig. 3 The antibacterial activity of tested bonding systems.

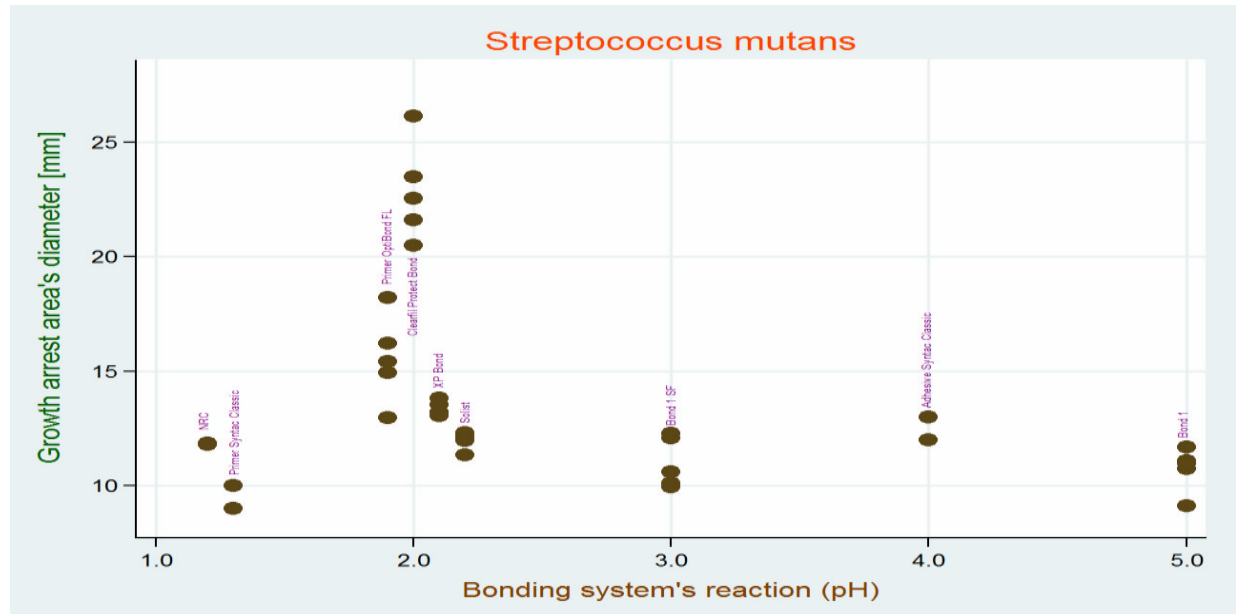

Fig. 4 The dependence of the $\mathrm{pH}$ value on an antibacterial activity against Streptococcus mutans. 


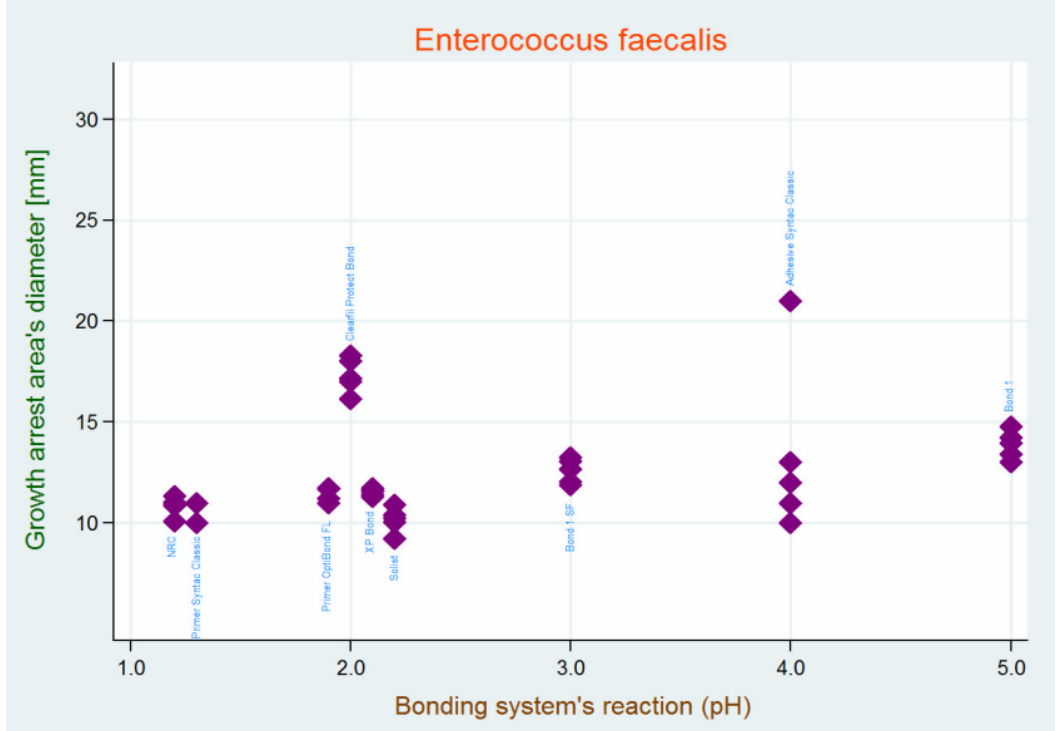

Fig. 5 The dependence of the $\mathrm{pH}$ value on an antibacterial activity against Enterococcus faecalis. 